\title{
A DEFINITION FOR STRONG RIESZIAN SUMMABILITY AND ITS RELATIONSHIP TO STRONG CESARO SUMMABIIITY
}

\author{
by A. V. BOYD and J. M. HYSLOP
}

(Received 30th October, 1950)

1. Introduction. Given a series $\sum_{n=0}^{\infty} a_{n}$, we define $A_{n}{ }^{(k)}, E_{n}{ }^{(k)}, k>-1$, by the relations

$$
A_{n}{ }^{(k)}=\sum_{\nu=0}^{n}\left(\begin{array}{c}
k+n-\nu \\
n-v
\end{array}\right) a_{\nu}, \quad E_{n}{ }^{(k)}=\left(\begin{array}{c}
k+n \\
n
\end{array}\right) .
$$

The series $\Sigma a_{n}$ is said to be summable $(C, k)$ to the sum $s$, if

$$
C_{n}{ }^{(k)}=A_{n}{ }^{(k)} / E_{n}{ }^{(k)} \rightarrow s
$$

as $n \rightarrow \infty$, and strongly summable $(C, k), k>0$, with index $p$, to the sum $s$, or summable $[C ; k, p]$ to the sum $s$, if

$$
\sum_{\nu=0}^{n}\left|C_{\nu}^{(k-1)}-s\right|^{p}=o(n)
$$

It is known * that necessary and sufficient conditions for $\Sigma a_{n}$ to be summable $[C ; k, p]$ $k>0, p \geqslant 1$, to the sum $s$ are that $\Sigma a_{n}$ be summable $(C, k)$ to the sum $s$ and that

$$
\sum_{\nu=0}^{n} \nu^{p}\left|a_{v}^{(k)}\right|^{p}=o(n)
$$

where $a_{v}{ }^{(k)}=C_{\nu}^{(k)}-C^{(k)}{ }_{\nu-1}$.

Defining $A_{k}(\omega), C_{k}(\omega)$ by the relations

$$
A_{k}(\omega)=\sum_{n<\omega}(\omega-n)^{k} a_{n}, \quad C_{k}(\omega)=\omega^{-k} A_{k}(\omega)
$$

we have the familiar definition that $\Sigma a_{n}$ is summable $(R, k)$ to the sum $s$ if $C_{k}(\omega) \rightarrow s$ as $\omega \rightarrow \infty$ continuously. If, in addition, we have

$$
\int_{1}^{\omega}\left|u \frac{d}{d u} C_{k}(u)\right|^{p} d u=o(\omega)
$$

it is then natural to say that $\Sigma a_{n}$ is strongly summable $(R, k)$, with index $p$, to the sum $s$, and write $\Sigma a_{n}$ is summable $[R ; k, p]$ to the sum $s$. In this definition it is assumed that $k>0, p \geqslant 1$.

It should be noted that, for the definition to be valid at all, it is necessary that $k p^{\prime}>1$, where $\frac{1}{p}+\frac{1}{p^{\prime}}=1$, since, writing $n=[u]$, we have $\uparrow$

whence

$$
\begin{aligned}
\left|u \frac{d}{d u} C_{k}(u)\right| & =k u^{-k}\left|\sum_{\nu=1}^{n}(u-v)^{k-1} \nu a_{\nu}\right| \\
& \geqslant k u^{-k}(u-n)^{k-1} n\left|a_{n}\right|-k u^{-k}\left|\sum_{\nu=1}^{n-1}(u-\nu)^{k-1} \nu a_{\nu}\right|,
\end{aligned}
$$

$$
\begin{aligned}
& k^{p} u^{-k p}(u-n)^{k p-p} n^{p}\left|a_{n}\right|^{p} \leqslant 2^{p}\left|u \frac{d}{d u} C_{k}(u)\right|^{p}+\left.\left.2^{p} k^{p} u^{-k p}\right|_{\nu=1} ^{n-1}(u-v)^{k-1} \nu a_{v}\right|^{p} \\
& \text { * J. M. Hyslop, Proc., Glasgow Math. Assoc., I., p. } 16 . \\
& \text { † See Lemma 2 below. }
\end{aligned}
$$


Now

$$
\begin{aligned}
\int_{1}^{\omega} u^{-k p}(u-n)^{k p-p} n^{p}\left|a_{n}\right|^{p} d u & \geqslant \sum_{n=1}^{[\omega]-1} \int_{n}^{n+1} u^{-k p}(u-n)^{k p-p} n^{p}\left|a_{n}\right|^{p} d u \\
& \geqslant \sum_{n=1}^{[\omega]}(n+1)^{-k p} n^{p}\left|a_{n}\right|^{p} \int_{n}^{n+1}(u-n)^{k \boldsymbol{p}-p} d u \\
& =\infty,
\end{aligned}
$$

unless $p(k-1)>-1$, that is, unless $k>1 / p^{\prime}$. Since

$$
\left.\left.\int_{1}^{\omega} u^{-k p}\right|_{\nu=1} ^{n-1}(u-v)^{k-1} v a_{\nu}\right|^{p} d u
$$

is finite, it follows that

$$
\int_{1}^{\omega}\left|u \frac{d}{d u} C_{k}(u)\right|^{p} d u
$$

is infinite unless $k p^{\prime}>1$.

Our object in this paper is to prove the following theorem :

THEOREM. If $k>0, p \geqslant 1, \frac{1}{p}+\frac{1}{p^{\prime}}=1, k p^{\prime}>1$, then summability $[C ; k, p]$ of the series $\Sigma a_{n}$ implies summability $[R ; k, p]$ of this series to the same sum, and conversely.

The proof of the theorem is based on several lemmas, most of which are well known.

2. Preliminary Lemmas. Lemia 1. If $* k>-1, \delta>0$, then

$$
A_{k+\delta}(\omega)=\frac{\Gamma(k+\delta+1)}{\Gamma(k+1) \Gamma(\delta)} \int_{0}^{\omega}(\omega-u)^{\delta-1} A_{k}(u) d u .
$$

Lemma 2. If $\dagger B_{k}(\omega)$ is the Rieszian sum of order $k$ for the series $\Sigma b_{n}$, where $b_{n}=n a_{n}$, then, for $k>0$,

$$
\omega^{k+1} \frac{d}{d \omega} C_{k}(\omega)=k B_{k-1}(\omega)=\frac{d}{d \omega} B_{k}(\omega) .
$$

LEMмA 3. We have, $\$$ for $k>-1$, the formal relations

$$
\begin{aligned}
& \sum_{n=0}^{\infty} A_{n}{ }^{(k)} x^{n}=(1-x)^{-k-1} \sum_{n=0}^{\infty} a_{n} x^{n} \\
& \sum_{n=0}^{\infty} n E_{n}{ }^{(k)} a_{n}{ }^{(k)} x^{n}=(1-x)^{-k} \sum_{n=0}^{\infty} n a_{n} x^{n}
\end{aligned}
$$

Lemma 4. If $\S 0<\theta \leqslant 1, k>0, q$ is any positive integer or zero and

then

$$
\gamma_{n, k}(\theta)=\sum_{\nu=0}^{n}(n+\theta-\nu)^{k-1} E_{\nu}{ }^{(-k-1)}
$$

where

$$
\gamma_{n, k}(\theta)=\delta(\theta) E_{n}^{(-k-1)}+\beta_{n, k},
$$

$$
\beta_{n, k}=O\left\{\sum_{\nu=0}^{n}(\nu+1)^{-k-1}(n-\nu+1)^{k-q-2}\right\}
$$

$$
\delta(\theta)=\theta^{k-1}+\sum_{r=0}^{q} e_{r} \theta^{r}
$$

and $e_{r}$ is independent of $n$ and $\theta$. When $k>1$ the term $\delta(\theta) E_{n}^{(-k-1)}$ may be incorporated in $\beta_{n, k}$, and $\theta$ may take the value zero.

* G. H. Hardy and M. Riesz, The General Theory of Dirichlet Series (Cambridge Tract, No. 18), 27.

† See, for example, J. M. Hyslop, Proc. Edinburgh Math. Soc., (2), 5 (1937), 46-54.

† E. Kogbetliantz, Bull. des Sciences Math., (2), 49 (1925), 234-56.

§. M. Hyslop, loc. cit. 
LEMMa 5. In the notation of Lemma 4, by suitable choice of $q$,

We have, if $q>2 k-1$,

$$
\beta_{n, k}=O\left\{(n+1)^{-k-1}\right\} \text {. }
$$

$$
\begin{aligned}
\beta_{n, k} & =O\left\{\sum_{0 \leqslant \nu \leqslant \frac{1}{2} n}(\nu+1)^{-k-1}(n-\nu+1)^{k-q-2}\right\}+O\left\{\sum_{\frac{1}{2} n \leqslant \nu \leqslant n}(\nu+1)^{-k-1}(n-\nu+1)^{k-q-2}\right\} \\
& =O\left\{\left(\frac{1}{2} n+1\right)^{k-q-2} \sum_{\nu=0}^{\infty}(\nu+1)^{-k-1}\right\}+O\left\{\left(\frac{1}{2} n+1\right)^{-k-1} \sum_{\nu=0}^{\infty}(\nu+1)^{k-q-2}\right\} \\
& =O\left\{(n+1)^{-k-1}\right\} .
\end{aligned}
$$

Lemma 6. If $*$ is a positive integer or zero, $A_{n}{ }^{(k)}$ can be expressed in the form

where $\alpha_{\rho}$ is independent of $n$.

$$
\sum_{\rho=0}^{k} \alpha_{\rho} A_{k}\left(n+\frac{\rho}{k}\right)
$$

Lemma 7. If $\alpha_{\nu} \geqslant 0, p \geqslant 1, \lambda>0, \lambda p^{\prime}>1$,

$$
\sum_{n=0}^{N}\left\{\sum_{\nu=0}^{n} \frac{\alpha_{\nu}}{(n-\nu+1)^{1+\lambda}}\right\}^{p} \leqslant K \sum_{n=0}^{N} \alpha_{n}^{p}
$$

where $K$ is independent of $N$.

When $p=1$ the result follows immediately on interchanging the order of summation. When $p>1$, we have, by Hölder's inequality,

since $\lambda p^{\prime}>1$. Hence

$$
\begin{aligned}
\left\{\sum_{\nu=0}^{n} \frac{\alpha_{\nu}}{(n-\nu+1)^{1+\lambda}}\right\}^{p} & =\left\{\sum_{\nu=0}^{n} \frac{\alpha_{\nu}}{n-\nu+1} \frac{1}{(n-\nu+1)^{\lambda}}\right\}^{p} \\
& \leqslant \sum_{\nu=0}^{n} \frac{\alpha_{\nu}{ }^{p}}{(n-\nu+1)^{p}} \cdot\left\{\sum_{\nu=0}^{n} \frac{1}{(n-\nu+1)^{\lambda p^{\prime}}}\right\}^{p / p^{\prime}} \\
& \leqslant K_{1} \sum_{\nu=0}^{n} \frac{\alpha_{\nu}{ }^{p}}{(n-\nu+1)^{p}}
\end{aligned}
$$

$$
\begin{aligned}
\sum_{n=0}^{N}\left\{\sum_{\nu=0}^{n} \frac{\alpha_{\nu}}{(n-\nu+1)^{1+\lambda}}\right\}^{p} & \leqslant K_{1} \sum_{n=0}^{N} \sum_{\nu=0}^{n} \frac{\alpha_{\nu}^{p}}{(n-\nu+1)^{p}} \\
& =K_{1} \sum_{\nu=0}^{N} \alpha_{\nu}^{p} \sum_{n=v}^{N} \frac{1}{(n-\nu+1)^{p}} \\
& \leqslant K \sum_{\nu=0}^{N} \alpha_{\nu}{ }^{p},
\end{aligned}
$$

since $p>1$.

3. Summability $[C ; k, p]$ implies summability $[R ; k, p]$. Since summability $(C, k)$ implies summability $(R, k)$ to the same sum, it is sufficient to show that, for $k>0, p \geqslant 1$, $k p^{\prime}>1$,

implies that

$$
\sum_{\nu=0}^{n}\left|\nu a_{\nu}{ }^{(k)}\right|^{p}=o(n)
$$

$$
\int_{1}^{X}\left|\omega \frac{d}{d \omega} C_{k}(\omega)\right|^{p} d \omega=o(X)
$$

* E. W. Hobson, The Theory of Functions of a Real Variable, II (1926), 93. 
By Lemmas 2 and 3 , if $N=[\omega]$,

$$
\begin{aligned}
\frac{d}{d \omega} C_{k}(\omega) & =k \omega^{-k-1} B_{k-1}(\omega) \\
& =k \omega^{-k-1} \sum_{n=0}^{N}(\omega-n)^{k-1} n a_{n} \\
& =k \omega^{-k-1} \sum_{n=0}^{N}(\omega-n)^{k-1} \sum_{\nu=0}^{n} E_{n-\nu}^{(-k-1)} \nu E_{\nu}^{(k)} a_{\nu}(k)
\end{aligned}
$$

Write $\omega=N+\theta, 0<\theta \leqslant 1$, and $n-\nu=\mu$. Then, interchanging the order of summation,

$$
\begin{aligned}
\omega \frac{d}{d \omega} C_{k}(\omega) & =k \omega^{-k} \sum_{\nu=0}^{N} \nu E_{\nu}{ }^{(k)} a_{\nu}{ }^{(k)} \stackrel{N=0}{N=\nu}_{\mu=0}(N+\theta-\nu-\mu)^{k-1} E_{\mu}{ }^{(-k-1)} \\
& =k \omega^{-k} \sum_{\nu=0}^{N} \nu E_{\nu}{ }^{(k)} a_{\nu}{ }^{(k)} \gamma_{N-\nu, k}(\theta) .
\end{aligned}
$$

Hence, by Lemmas 4 and 5 ,

$$
\begin{aligned}
\left|\omega \frac{d}{d \omega} C_{k}(\omega)\right|^{p} & =O\left[\omega^{-k p}\left\{\sum_{\nu=0}^{N} \nu E_{\nu}{ }^{(k)}\left|a_{\nu}{ }^{(k)}\right|(\omega-N)^{k-1}\left|E_{N-\nu}{ }^{(-k-1)}\right|\right\}^{p}\right] \\
& +O\left[\omega^{-k}\left\{\sum_{\nu=0}^{N} \nu E_{\nu}{ }^{(k)}\left|a_{\nu}{ }^{(k)}\right| \sum_{r=0}^{q}\left|e_{r}\right| \theta^{r}\left|E_{N-\nu}{ }^{(-k-1)}\right|\right\}^{p}\right] \\
& +O\left[\omega^{-k p}\left\{\sum_{\nu=0}^{N} \nu E_{\nu}{ }^{(k)}\left|a_{\nu}{ }^{(k)}\right|(N-\nu+1)^{-k-1}\right\}^{p}\right],
\end{aligned}
$$

and, since $E_{N-\nu}{ }^{(-k-1)}=O\left\{(N-\nu+1)^{-k-1}\right\}, 0<\theta \leqslant 1$, the result will follow if we show that the two integrals *

$$
\begin{aligned}
& \int_{1}^{X}(\omega-N)^{p(k-1)}\left\{\omega^{-k} \sum_{\nu=1}^{N} \frac{\nu^{k} \nu\left|a_{\nu}{ }^{(k)}\right|}{(N-\nu+1)^{1+k}}\right\}^{p} d \omega, \\
& \int_{1}^{X}\left\{\omega^{-k} \sum_{\nu=1}^{N} \frac{\nu^{k} \cdot \nu\left|a_{\nu}{ }^{(k)}\right|}{(N-\nu+1)^{1+k}}\right\}^{p} d \omega
\end{aligned}
$$

are each $o(X)$.

The second integral is not greater than

$$
\begin{aligned}
\int_{1}^{X}\left\{\sum_{\nu=1}^{N} \frac{\nu\left|a_{\nu}{ }^{(k)}\right|}{(N-\nu+1)^{1+k}}\right\}^{p} d \omega & \leqslant \sum_{N=1}^{[X]} \int_{N}^{N+1} \int_{\left\{\sum_{\nu=1}^{N} \frac{\nu\left|a_{\nu}{ }^{(k)}\right|}{(N-\nu+1)^{1+k}}\right\}^{p} d \omega} \\
& =\sum_{N=1}^{[X]}\left\{\sum_{\nu=1}^{N} \frac{\nu\left|a_{\nu}{ }^{(k)}\right|}{(N-\nu+1)^{1+k}}\right\}^{p} \\
& =O\left\{\left.\sum_{N=1}^{[X]} \nu a_{\nu}{ }^{(k)}\right|^{p}\right\}=o(X)
\end{aligned}
$$

by hypothesis and Lemma 7. The first integral is not greater than

$$
\begin{aligned}
\int_{1}^{X}(\omega-N)^{p k-p}\left\{\sum_{\nu=1}^{N} \frac{\nu\left|a_{\nu}^{(k)}\right|}{(N-\nu+1)^{1+k}}\right\}^{p} d \omega & \leqslant \sum_{N=1}^{[X]}\left\{\sum_{\nu=1}^{N} \frac{\nu\left|a_{\nu}^{(k)}\right|}{(N-\nu+1)^{1+k}}\right\}^{p} \int_{N}^{N+1}(\omega-N)^{k p-p} d \omega \\
& \leqslant K \sum_{N=1}^{[X]}\left\{\sum_{\nu=1}^{N} \frac{\nu\left|a_{\nu}^{(k)}\right|}{(N-\nu+1)^{1+k}}\right\}^{p},
\end{aligned}
$$

since $k p^{\prime}>1$. It now follows as above that this integral is also $o(X)$.

* The term $v=0$ in each of the preceding expressions is, of course, zero, and may therefore be omitted. 
4. Summability $[R ; k, p]$ implies summability $[C ; k, p]$.

Since summability $(R, k)$ implies summability $(C, k)$ to the same sum, it is sufficient to show that, for $k>0, p \geqslant 1, k p^{\prime}>1$,

implies that

$$
\int_{1}^{X}\left|\omega \frac{d}{d \omega} C_{k}(\omega)\right| d \omega=o(X)
$$

We have, from Lemma 3,

$$
\sum_{n=1}^{N}\left|n a_{n}^{(k)}\right|^{p}=o(N)
$$

$$
\begin{aligned}
n E_{n}{ }^{(k)} a_{n}{ }^{(k)} & =\sum_{\nu=0}^{n} E_{n-\nu}{ }^{(k-1)} b_{\nu} \\
& =\sum_{\nu=0}^{n} E_{n-\nu}{ }^{(k-1)} \sum_{\mu=0}^{\nu} E_{\nu-\mu}{ }^{(-i-2)} B_{\mu}{ }^{(i)},
\end{aligned}
$$

where $i$ is the integer next greater than $k$. From Lemmas 1, 2 and 6 , it then follows that

$$
\begin{aligned}
n E_{n}{ }^{(k)} a_{n}{ }^{(k)} & =\sum_{\rho=0}^{i} d_{\rho} \sum_{\nu=0}^{n} E_{n-\nu}{ }^{(k-1)} \sum_{\mu=0}^{\nu} E_{\nu-\mu}{ }^{(-i-2)} B_{i}(\mu+\phi) \\
& =D_{k} \sum_{\rho=0}^{i} d_{\rho} \sum_{\nu=0}^{n} E_{n-\nu}{ }^{(k-1)} \sum_{\mu=0}^{\nu} E_{\nu-\mu}{ }^{(-i-2)} \int_{0}^{\mu+\phi}(\mu+\phi-u)^{i-k} \frac{d}{d u} B_{k}(u) d u,
\end{aligned}
$$

where $\phi=\phi(\rho)=\rho / i$ and

$$
D_{k}=\frac{\Gamma(i+1)}{\Gamma(k+1) \Gamma(1+i-k)} \cdot
$$

We now obtain, from Lemma 2 ,

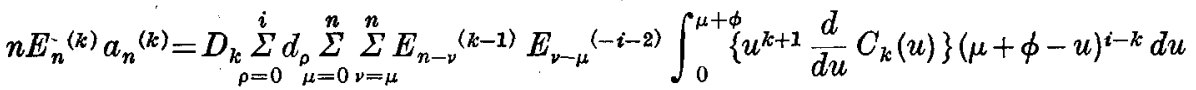

$$
\begin{aligned}
& =D_{k} \sum_{\rho=0}^{i} d_{\rho} \sum_{\mu=0}^{n} \int_{0}^{\mu+\phi}(\mu+\phi-u)^{i-k} u^{k+1} \frac{d}{d u} C_{k}(u) d u \sum_{\nu=\mu}^{n} E_{n-\nu}{ }^{(k-1)} E_{\nu-\mu}{ }^{(-i-2)} .
\end{aligned}
$$

But

$$
\sum_{\nu=\mu}^{n} E_{n-\nu}{ }^{(k-1)} E_{\nu-\mu}{ }^{(-i-2)}=\sum_{8=0}^{n-\mu} E_{n-\mu-s}{ }^{(k-1)} E_{s}^{(-i-2)},
$$

which is the coefficient of $x^{n-\mu}$ in the expansion of $(1-x)^{-k}(1-x)^{i+1}$, and is therefore equal to $E_{n-\mu}{ }^{(k-i-2)} \quad$ Hence, since $0 \leqslant \phi \leqslant 1$, we have

$$
\begin{aligned}
n\left|a_{n}{ }^{(k)}\right| & =O\left\{n^{-k} \sum_{\rho=0}^{i} \int_{0}^{n+\phi} u^{k+1}\left|\frac{d}{d u} C_{k}(u)\right| d u\left|\sum_{\mu=[u-\phi]}^{n}(\mu+\phi-s)^{i-k} E_{n-\mu}{ }^{(k-i-2)}\right|\right\} \\
& =O\left\{n^{-k} \sum_{\rho=0}^{i} \sum_{s=0}^{n} \int_{s}^{s+1} u^{k+1}\left|\frac{d}{d u} C_{k}(u)\right| d u\left|\sum_{\mu=8}^{n}(\mu+\phi-s)^{i-k} E_{n-\mu}{ }^{(k-i-2)}\right|\right\} .
\end{aligned}
$$

The innermost sum in the expression is

$$
\sum_{\lambda=0}^{n-s}(n-s-\lambda+\phi)^{i-k} E_{\lambda}^{(k-i-2)}=\gamma_{n-s, i+1-k}(\phi),
$$

which, by Lemmas 4 and 5 , is

$$
O\left\{(n-s+1)^{k-i-2}\right\}, \quad(\rho=0,1,2, \ldots, i) .
$$

Writing

$$
\alpha_{s}=\int_{8}^{s+1}\left|u \frac{d}{d u} C_{k}(u)\right| d u
$$


it follows that

$$
\begin{aligned}
n\left|a_{n}{ }^{(k)}\right| & =O\left\{n^{-k} \sum_{s=0}^{n}(n-s+1)^{k-i-2} \int_{s}^{s+1} u^{k}\left|u \frac{d}{d u} C_{k}(u)\right| d u\right\} \\
& =O\left\{\sum_{s=0}^{n} \alpha_{s}(n-s+1)^{k-i-2}\right\},
\end{aligned}
$$

and, by Lemma 7, that, for $p \geqslant 1$,

$$
\begin{aligned}
\sum_{n=1}^{N}\left|n a_{n}{ }^{(k)}\right|^{p} & =O\left\{\sum_{s=1}^{N} \alpha_{s}{ }^{p}\right\} \\
& =O\left\{\sum_{s=1}^{N}\left(\int_{s}^{s+1} u\left|\frac{d}{d u} C_{k}(u)\right| d u\right)^{p}\right\} \\
& =O\left\{\sum_{s=1}^{N}\left(\int_{s}^{s+1}\left|u \frac{d}{d u} C_{k}(u)\right| d u\right)\left(\int_{s}^{s+1}\left(1^{p^{\prime}} d u\right)^{p / p^{\prime}}\right\}\right. \\
& =O\left\{\int_{1}^{N+1}\left|u \frac{d}{d u} C_{k}(u)\right|^{p} d u\right\}=o(N)
\end{aligned}
$$

The theorem is therefore completely proved.

UNIVERSITY OF THE WITWATERSRAND,

JOHANNESBURG. 